\title{
Streptomyces turgidiscabies Secretes a Novel Virulence Protein, Nec1, Which Facilitates Infection
}

\author{
Madhumita Joshi, ${ }^{1}$ Xin Rong, ${ }^{1}$ Simon Moll, ${ }^{1,2}$ Johan Kers, ${ }^{1}$ Christopher Franco, ${ }^{2}$ and Rosemary Loria ${ }^{1}$ \\ ${ }^{1}$ Department of Plant Pathology, Cornell University, Ithaca, NY 14853-5904, U.S.A.; ${ }^{2}$ Department of Medical Biotechnology, \\ School of Medicine, Flinders University, Bedford Park, SA 5042, Australia
}

Submitted 27 September 2006. Accepted 19 December 2006.

Emergence of new, economically important plant-pathogenic species in the mostly saprophytic genus Streptomyces involves acquisition of a large, mobile pathogenicity island (PAI). Biosynthetic genes for a phytotoxin, thaxtomin A, are contained on this PAI. The Nec1 protein has necrogenic activity on excised potato tuber tissue, and the encoding gene is highly conserved in plant-pathogenic Streptomyces spp. The $\mathrm{G}+\mathrm{C}$ content of nec1 indicates lateral transfer from an unrelated taxon; however, the nucleic acid and protein databases have not yielded homologs. Data presented in this article demonstrate that the Nec1 protein is necrogenic when expressed in Escherichia coli and that an active 16-kDa form of Nec1 is secreted from the plant pathogen Streptomyces turgidiscabies. Deletion analysis of nec1 demonstrated that the 151 -amino-acid C-terminal region of the Nec1 protein is sufficient to confer necrogenic activity. Analysis of nec1 transcriptional start sites indicates that two mRNA species are produced and that the site of transcription initiation is influenced by glucose. $S$. turgidiscabies containing a nec1 deletion was greatly compromised in virulence on Arabidopsis thaliana, Nicotiana tabacum, and Raphanus sativus seedlings. The wild-type strain, $S$. turgidiscabies Car8, aggressively colonized and infected the root meristem of radish, whereas the $\Delta n e c 1$ mutant Car811 did not. Taken together, these data suggest that Nec1 is a secreted virulence protein with a conserved plant cell target that acts early in plant infection.

Additional keywords: plant defense, potato scab.

Members of the genus Streptomyces are gram-positive, filamentous prokaryotes that are best known for their ability to produce biologically active secondary metabolites, including antibiotics (Challis and Hopwood 2003). Most members of this genus are soil-inhabiting saprophytes and secrete an abundance of hydrolytic enzymes for degradation of plant- and animal-derived polymers (Belfaquih et al. 2002; Broadway et al. 1995). Streptomyces genomes have large coding capacities and flexible structures, consistent with an adaptive, resourceful bacterium (Bentley et al. 2002; Ikeda et al. 2003; Parajuli et al. 2004). Interestingly, some Streptomyces spp. have symbiotic relationships with higher eukaryotes. A few of these relationships appear to be beneficial to the eukaryote (Kaltenpoth et al. 2005; Tokala et al. 2002), whereas most result in disease

Corresponding author: R. Loria; E-mail: r121@ cornell.edu

Current address of J. Kers: Stanford University School of Medicine, Department of Genetics, Stanford, CA 94305-5120, U.S.A.
(Loria et al. 1997). Among the latter group are Streptomyces spp. that infect higher plants. The best characterized of these, Streptomyces scabies, S. acidiscabies, and S. turgidiscabies, are known for causing the economically important disease potato scab; however, these pathogens are neither host nor tissue specific. All of these species produce thaxtomins, members of a family of nitrated dipeptide phytotoxins (King et al. 1989, 1996). Nitration of the nonribosomally synthesized peptide (Healy et al. 2000) occurs via a nitric oxide synthase (Kers et al. 2004). Thaxtomins are required for disease development by the diverse Streptomyces spp. that cause potato scab (Healy et al. 2000). Production of thaxtomin in media requires the presence of plant cell wall components, and media containing oat bran is particularly effective in inducing toxin production (Goyer et al. 1998). Thaxtomin has a necrogenic phenotype on excised potato tuber tissue (EPT) and induces cell hypertrophy, binucleate cells, and abnormal cell plates in onion root tip cells at nanomolar concentrations (Fry and Loria 2002). A direct or indirect target of thaxtomin is cellulose biosynthesis, accounting for the cell swelling phenotype in expanding tissue (Scheible et al. 2003).

The genes for thaxtomin biosynthesis lie on a large (660$\mathrm{kb}$ ), mobilizable pathogenicity island (PAI) that is involved in the emergence of new plant-pathogenic species in agricultural systems (Kers et al. 2005). The virulence gene necl is also located on the PAI, approximately $325 \mathrm{~kb}$ from the thaxtomin biosynthetic genes in $S$. turgidiscabies. The necl gene was discovered through expression of an S. scabies cosmid library in the nonpathogen $S$. lividans; the cosmid carrying necl had a necrogenic phenotype on EPT (Bukhalid and Loria 1997). The necl gene has a low $\mathrm{G}+\mathrm{C}$ content that is atypical of Streptomyces genomic DNA and the surrounding PAI sequences (Kers et al. 2005). The low $\mathrm{G}+\mathrm{C}$ content of necl indicates lateral transfer from an unrelated taxon; however, the nucleic acid and protein databases have not yielded homologs. Thus, necl appears to be a novel virulence gene. The sequence of necl is identical across multiple strains of $S$. scabies, $S$. acidiscabies, and $S$. turgidiscabies (Bukhalid et al. 2002), indicating that it was introduced onto the PAI prior to its mobilization into these strains and relatively recently in evolutionary time.

The necrogenic phenotype of the protein encoded by the necl gene, its presence on the PAI, and its conservation in pathogenic Streptomyces spp. suggests a role in host-pathogen interactions. However, because of the lack of necl homologs, it has been difficult to develop a hypothesis as to the role of necl in virulence. Here, we show that necl encodes a virulence protein that is secreted via N-terminal processing. We also demonstrate that necl confers a dramatic virulence phenotype on Nicotiana tabacum (tobacco), Raphanus sativus (radish), and Arabidopsis thaliana (Arabidopsis). Therefore, 
Nec1, like thaxtomin, has a conserved plant cell target, consistent with the lack of host specificity of plant-pathogenic streptomycetes.

\section{RESULTS}

The nec1 gene encodes a secreted, necrogenic protein.

Previous studies demonstrated that expression of necl in $S$. lividans was sufficient to allow this nonpathogen to necrotize and colonize EPT, and produce limited necrosis on immature whole tubers (Bukhalid and Loria 1997). The necrogenic factor was found in the supernatant of cultures of S. lividans expressing necl and was not extractable with organic solvents, as is thaxtomin. These data suggested that the necl gene might encode a necrogenic, secreted protein. To test the hypothesis that the Nec1 protein is the necrogenic factor, we cloned the 663-bp nec1 open reading frame (ORF) into pET28a, resulting in pET::nec1, and expressed it in Escherichia coli BLR(DE3). Sodium dodecyl sulfate polyacrylamide gel electrophoresis (SDS-PAGE) analysis revealed a band of approximately the expected size $(24.15 \mathrm{kDa})$, making up approximately $10 \%$ of the total proteins extracted from both the supernatant and cell pellets of induced cultures. An additional protein band of approximately $16 \mathrm{kDa}$ in size accumulated in the cell pellet. Immunoblot analysis, using a rabbit polyclonal antibody raised against the $24.15-\mathrm{kDa} \mathrm{Nec} 1$ protein, confirmed that both the 16- and 24.15-kDa bands were Nec1 (Fig. 1). Cultures of $E$. coli $\mathrm{BLR}(\mathrm{DE} 3)$ carrying pET::necl (full-length necl cloned in pET28a) induced by IPTG, caused necrosis on EPT (Fig. 1). However, cultures of E. coli BLR(DE3) containing the empty plasmid vector did not (Fig. 1). The necrogenic activity of a crude protein preparation from E. coli BLR (DE3) pET::nec1 was abolished by proteinase and heat treatments (data not presented), consistent with the hypothesis that the Nec1 protein is responsible for the necrogenic activity on EPT.

In order to determine whether Nec1 is a secreted virulence protein, the wild-type strain $S$. turgidiscabies Car8 and an $S$. turgidiscabies $\Delta$ nec1 mutant (Car811) were grown for 15, 20, or $24 \mathrm{~h}$ in mannitol soy flour (MSF) medium. These timepoints were chosen because preliminary observations had indicated that Nec1 was produced prior to thaxtomin. Cells were separated from culture supernatant by centrifugation and proteins were precipitated from both the culture supernatant and cell pellet. Crude protein prepared from supernatants and pellets was assessed for necrotization in the EPT assay. These protein preparations also were separated using SDS-PAGE and evaluated for the presence of the Nec1 protein by Western analysis. Beginning at $20 \mathrm{~h}$, a $16-\mathrm{kDa} \mathrm{Nec} 1$ band was detected in the supernatant of $S$. turgidiscabies Car8 cultures. The Nec1 protein was absent from the supernatant of Car811 and the cell
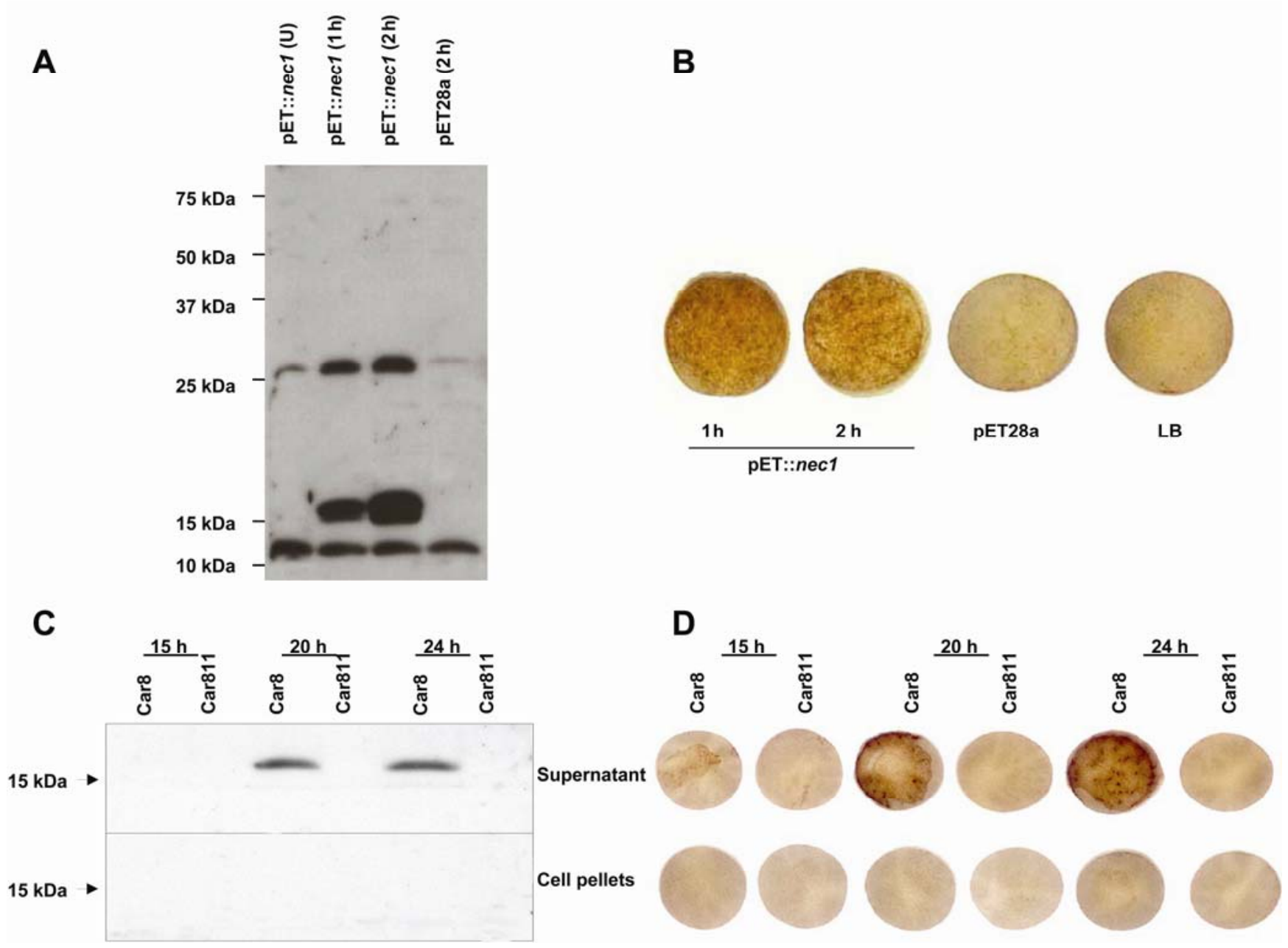

Fig. 1. Western analysis, using a rabbit polyclonal antibody raised against the 24.15-kDa Nec1 protein, and necrogenic activity of the Nec1 protein expressed in Streptomyces turgidiscabies and Escherichia coli.. A, Western analysis of protein from E. coli BLR(DE3) cultures expressing pET::nec1 or the vector control. Cultures were induced for 1 or $2 \mathrm{~h}$ or uninduced (U). B, Necrogenic activity of the protein extracts from the cultures described in A, in the excised potato tuber assay (EPT). C, Western analysis of protein extracts from the supernatant and cell pellet of the wild-type strain S. turgidiscabies Car8 and the $\Delta$ nec1 mutant Car811 at three timepoints. D, Necrogenic activity of protein extracts described in C in the EPT assay. 
pellet from both strains at all timepoints (Fig. 1). Necrosis of EPT occurred in those fractions in which Nec1 was identified by Western analysis, confirming that the protein was biologically active after secretion (Fig. 1).

\section{The nec1 gene has two transcriptional start sites.}

The necl gene originally was defined as a 663-bp ORF encoding a 221-amino-acid protein with a predicted size of approximately $24.15 \mathrm{kDa}$ (Bukhalid et al. 1998). Western analysis of the necl product in the supernatant of S. turgidiscabies suggested that the encoded protein could be secreted via a mechanism that involves cleavage of an N-terminal signal peptide to produce a $16-\mathrm{kDa}$ mature protein. Both the Sec and Tat protein secretion systems exist in Streptomyces spp. and involve N-terminal processing of secretion signals; therefore, Nec1 could be targeted to either secretion pathway (Gauthier et al. 2005). The necl gene contains three possible translational start codons (ATG-1, GTG, and ATG-2) (Fig. 2). Therefore, to facilitate bioinformatics analysis of the Nec1 secretion pathway and prediction of the $\mathrm{N}$-terminal cleavage site, we mapped the transcriptional start site of necl. Total RNA was isolated from $S$. turgidiscabies Car8 grown in oat bran broth (OBB) with or without glucose; OBB supports thaxtomin production that is catabolically suppressed by the addition of glucose. We used rapid amplification of cDNA ends (RACE) to identify two partial 5' transcripts (237 and $177 \mathrm{bp}$ ) in the presence of glucose; their sequences corresponded to the transcriptional start sites T1 and T2, respectively (Figs. 2 and 3). Interestingly, only the T2 site was used in the absence of glucose. These results were confirmed with $\mathrm{S} 1$ nuclease mapping (data not presented). The unique transcriptional start site, $\mathrm{T} 1$, is lo-

\section{GACGCAACCA CGGCGGGAGC GAACTGCTAG CACTTCAGAT CGCCAATCGC CTCAGCCATT CGGCCAGACT GCGGGTCAAG GTCTGCAGGT TTCTGGCCTT TCGGTCGAGG TGCCCACGCC GAGACAGCGC GAAGATGAGC GCGAACGGAA GCCCCGGACG TAACGGGGGC ${ }^{-35}$ CAACCAGTGC TTGGGCCGGT ATGCTTCTTC $\stackrel{\text { T2 }}{\longrightarrow}$ \\ CTGAAAGEGC TTTAAAAC ACCTCGCCTG CAGAGAGGAC GTGTAGTGA \\ ATCTGAAAATT CGCACTCTTG GAGATCTCAT GCGAACCTCT GGAGTGACG CCAAAGACTCA ATCAAGTTCG CCAAAGC}

Fig. 2. Nucleotide sequence of the necl promoter and partial coding region used for $\mathrm{S} 1$ nuclease mapping and the rapid amplification of cDNA ends study. The transcription start sites (T1 and T2) are shown by arrows, putative -10 sequences are indicated with a broken underline (T1) or a double underline (T2), and -35 sequences of both the transcriptional start sites are indicated with double underlines. Three predicted translation start sites are in bold font. The two tandem repeats flanking T1 are underlined with single lines. The predicted binding site for transcription factor rpoD17 is boxed.

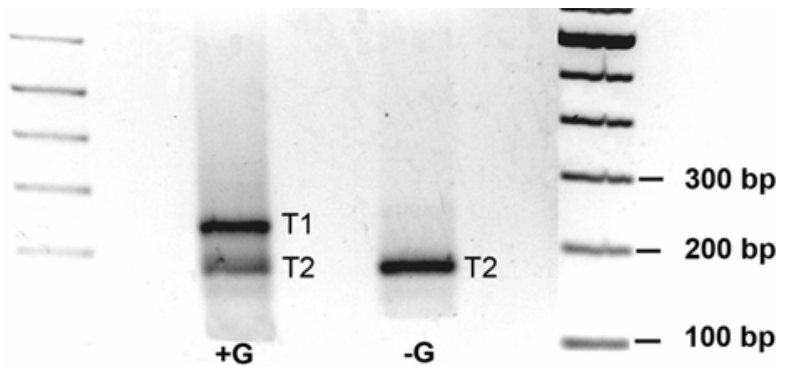

Fig. 3. Gel electrophoresis of polymerase chain reaction products generated by rapid amplification of cDNA ends (RACE) kit. cDNA were synthesized from $340 \mathrm{ng}$ of total RNA extracted from the Car8 culture grown in oat bran broth medium with $(+\mathrm{G})$ or without $(-\mathrm{G})$ glucose. The $5^{\prime}$ end of the nec 1 cDNA was isolated by using RACE. Two transcripts of approximately 237 bp (T1) and approximately 177 bp (T2) were identified after sequencing of the amplified product. cated 103 bp upstream of the second translational start codon (GTG) (Fig. 2). An 8-bp direct repeat (AGCGCGAA) overlaps T1. There is a putative -35 core promoter sequence (GNTTNC) $49 \mathrm{bp}$ upstream of T1. A putative streptomycete -10 consensus sequence (GAGACA) also was found (Strohl 1992) 16 bp upstream of T1. The conserved necl transcriptional start site, T2, is located $43 \mathrm{bp}$ upstream of the second putative translational start site (GTG) of necl (Fig. 2). Promoter regions were predicted at -10 (CGGTATGC) and -35 positions (GGGCCA) of $\mathrm{T} 2$, although the consensus sequence in the -35 region was weak. A binding site CGCCTTTT for the transcription factor rpoD17 (Robison et al. 1998) occurs 4 bp down stream of T2.

Bioinformatic analysis of the putative $\mathrm{N}$ terminal signal sequences of Nec1 predicted that the protein could be targeted to either the Sec pathway (Bendtsen et al. 2004) or the Tat pathway (Bendtsen et al. 2005). Because both $\mathrm{T} 1$ and $\mathrm{T} 2$ are downstream of the first translational start codon (ATG-1), we eliminated this start codon from further consideration (Fig. 4). Assuming that translation begins at the second start codon (GTG), Nec1 is predicted to be secreted through the Tat pathway (signal peptide probability 1.000) with the cleavage site at amino acids 83 to 84 (VVA-NP). However, secretion by the Sec pathway is also possible (signal peptide probability 0.911) with the predicted cleavage site at amino acids 90 to 91 (AFA-NS). If the third translational start codon is used (ATG-2), the signal peptide probability (0.911) and cleavage site amino acids 90 to 91 (AFA-NS) for both the Tat and Sec secretion pathway are the same (Fig. 4).

Because Nec1 lacks homologs or recognizable motifs, we conducted deletion analysis to identify regions of the protein responsible for necrogenic activity. The necl deletions were constructed in pET28a and expressed in E. coli strain BLR(DE3) (Novagen, Madison, WI, U.S.A.). Protein expression was induced with IPTG and proteins were precipitated from whole cultures. The sizes of the truncated Nec1 proteins were confirmed with SDS-PAGE and necrogenic activity was evaluated in the EPT assay. The full-length Nec1 protein (amino acids 1 to 221) and constructs containing amino acids 38 to 221 and 71 to 221 retained their ability to necrotize po-

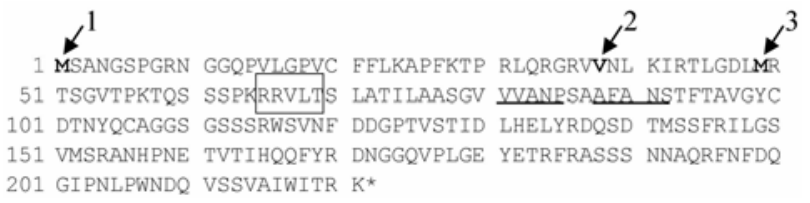

Fig. 4. Amino acid sequence of the full-length Nec1 protein, showing three possible translational start sites (arrows). Nec1 is predicted to be secreted through both the twin arginine (Tat) and Sec secretion pathways; the two putative cleavage sites are underlined. The predicted Tat site is boxed. An asterisk $(*)$ represents a stop codon.

Table 1. Full-length and deletion constructs of necl and their necrogenic activity $^{\mathrm{a}}$

\begin{tabular}{|c|c|c|}
\hline Constructs & Length (aa) & Symptoms \\
\hline pET::necl (full-length necl ORF) & 1 to 221 & + \\
\hline pET::nec1_38-221 ${ }^{\mathrm{c}}$ & 38 to 221 & + \\
\hline pET::nec1_71-221 & 71 to 221 & + \\
\hline pET::nec1_81-221 & 81 to 221 & - \\
\hline pET::nec1_91-221 & 91 to 221 & - \\
\hline pET::nec1_101-221 & 101 to 221 & - \\
\hline pET::nec1_38-186 & 38 to 186 & - \\
\hline pET::nec1_71-186 & 71 to 186 & - \\
\hline
\end{tabular}

a Prepared using pET28a vector.

${ }^{\mathrm{b}}$ Symptoms present (+) or absent (-) on potato tuber slice.

c Deletion constructs are parts of the full-length necl open reading frame (ORF). 
tato tissue, while constructs encoding amino acids 81 to 221 , 91 to 221,101 to 221,38 to 186 , and 71 to 186 were inactive in the EPT assay (Table 1). These data are consistent with $\mathrm{N}$ terminal processing of Nec1 and indicate that the C-terminal portion of Nec1 (amino acids 71 to 221) is critical for activity.

The nec1 gene has a virulence phenotype on Arabidopsis, tobacco, and radish.

Because $S$. scabies and other potato scab-causing streptomycetes are known to infect roots of many plant species (Hooker 1949; Leiner et al. 1996), we investigated the role of necl in pathogenicity of S. turgidiscabies on the model plants Arabi-

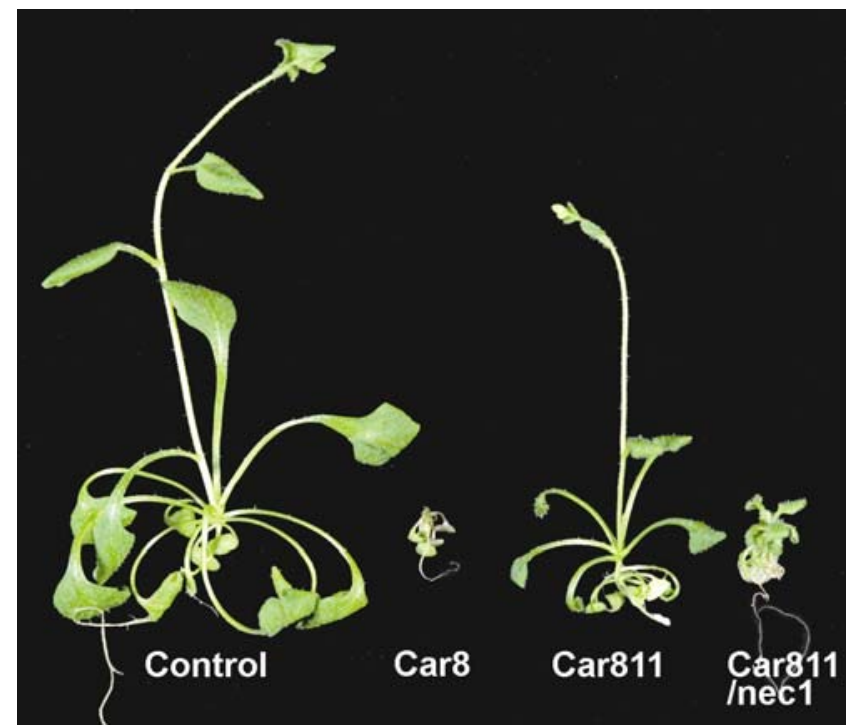

Fig. 5. Arabidopsis plants 3 weeks after inoculation with Streptomyces turgidiscabies strains. Seed were grown in sterile containers on Murashige and Skoog agar medium containing $2 \%$ sucrose. Plants were inoculated by adding spores (approximately $10^{6}$ ) to the surface of the medium on which the seedlings were growing, 7 days after seeding. Treatments were: wildtype strain S. turgidiscabies Car8, the $\Delta$ necl mutant S. turgidiscabies Car811, or the Car811 strain complemented with pSET152::nec1, in which necl is expressed in trans under its native promoter. dopsis and tobacco and on the economic host radish. Seedlings were grown in sterile containers on Murashige and Skoog medium (Murashige and Skoog 1962) containing 2\% sucrose. Plants were inoculated by adding spores (approximately $10^{6}$ ) to the surface of the medium on which the seedlings were growing. Treatments were the wild-type strain $S$. turgidiscabies Car8, the $\Delta$ nec1 mutant $S$. turgidiscabies Car811, or the Car811 strain complemented with pSET152::nec1, in which necl is expressed in trans under its native promoter. Arabidopsis plants inoculated with $S$. turgidiscabies Car8 were severely stunted and necrotic; most plants died in 14 to 20 days after inoculation, demonstrating that this wild-type strain is an aggressive pathogen of the model plant Arabidopsis (Fig. 5). In contrast, noninoculated plants grew vigorously and had healthy root systems. Plants infected with the $\Delta n e c 1$ mutant, $S$. turgidiscabies Car811, were intermediate in size, relative to control plants and those inoculated with the wild-type pathogen (Fig. 5). Disease severity also was intermediate; root necrosis was minimal and plant mortality was 10 to $15 \%$. The difference in disease severity and plant growth between wild-type and $\Delta n e c l$ strains demonstrate that necl is a virulence factor on Arabidopsis. The necl virulence phenotype was partially restored in Arabidopsis plants inoculated with the complementation strain S. turgidiscabies Car811 (pSET152::nec1). Plant growth suppression and disease severity were reduced compared with the wild-type strain but were much greater than for the $\Delta$ necl mutant (Fig. 5), confirming that necl is a virulence factor. Virulence phenotypes on radish were very similar to those on Arabidopsis in these studies (data not shown).

The wild-type strain $S$. turgidiscabies Car8 caused shoot and root stunting on tobacco within 2 to 3 weeks after inoculation. The most dramatic symptom was the production of gall-like structures at the tips of lateral roots upon infection. Root tips became necrotic and then, within 2 to 3 weeks, expanded into terminal, necrotic, gall-like structures (Fig. 6). The plants infected with the $\Delta n e c 1$ mutant had reduced root growth and produced gall-like structures at root tips; however, these structures were small and only slightly necrotic. Rather than being terminal structures, they produced roots and shoots 4 weeks after inoculation (Fig. 6). Roots and shoots emerging from the gall-like structures produced on $\Delta n e c 1$-infected plants contin-
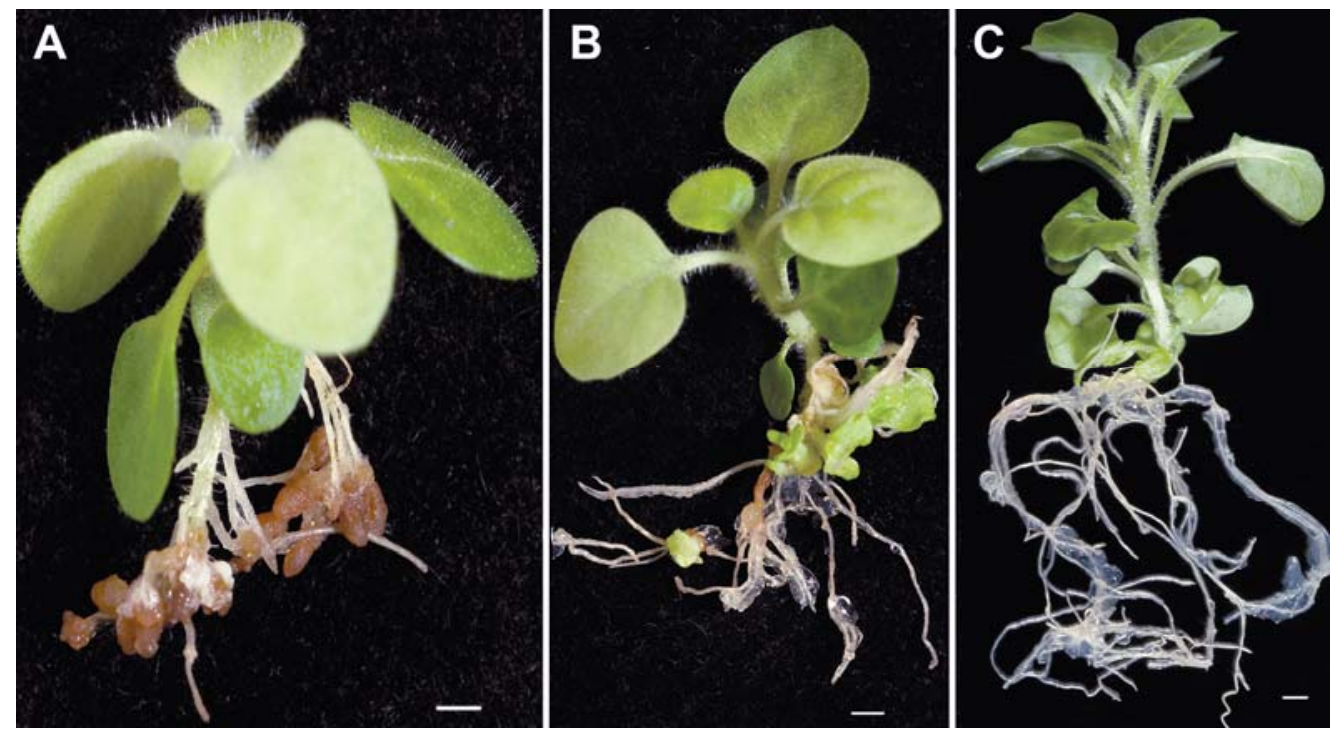

Fig. 6. Tobacco plants 3 weeks after inoculation with Streptomyces turgidiscabies strains. Seed were grown in sterile containers on Murashige and Skoog agar medium containing $2 \%$ sucrose. Plants were inoculated by adding spores (approximately $10^{6}$ ) to the surface of the medium on which the seedlings were growing, 7 days after seeding. Treatments were A, wild-type strain S. turgidiscabies Car8; B, the $\Delta$ necl mutant S. turgidiscabies Car811; or C, the noninoculated control. Bars represent $1 \mathrm{~mm}$. 
ued to grow without noticeable disease symptoms. Uninoculated control plants had a vigorous root system, were larger than inoculated plants, and lacked gall-like structures.

These observations suggested that there is a qualitative difference in the way $S$. turgidiscabies Car8 and the $\Delta n e c 1 \mathrm{mu}-$ tant interact with plant roots. Therefore, we used transmission and confocal scanning microscopy with green fluorescent protein (GFP)-labeled S. turgidiscabies Car8 and S. turgidiscabies Car811 to observe colonization of roots and plant morphological responses. Radish plants grown in a hydroponic system were used in this study to facilitate plant handling and microscopy. The wild-type strain aggressively colonized the root tip, resulting in epiphytic, intra- and intercellular growth of the wild-type strain within 7 days (Fig. 7). The region of cell differentiation contained an abundance of root hairs and was greatly swollen in plants inoculated with the wild-type strain (Fig. 7); swelling was due to the dramatic radial expansion of cells (Fig. 7). Roots inoculated with the $\Delta n e c 1$ mutant were slightly swollen, relative to the control, apparently due to a slight increase in cell size. The number of root hairs in the zone of differentiation was intermediate between the wild-type and control treatments. The $\Delta$ necl mutant did not aggressively colonize the root tip and seems to be restricted to the root cap; intra- and intercellular growth was absent and epiphytic

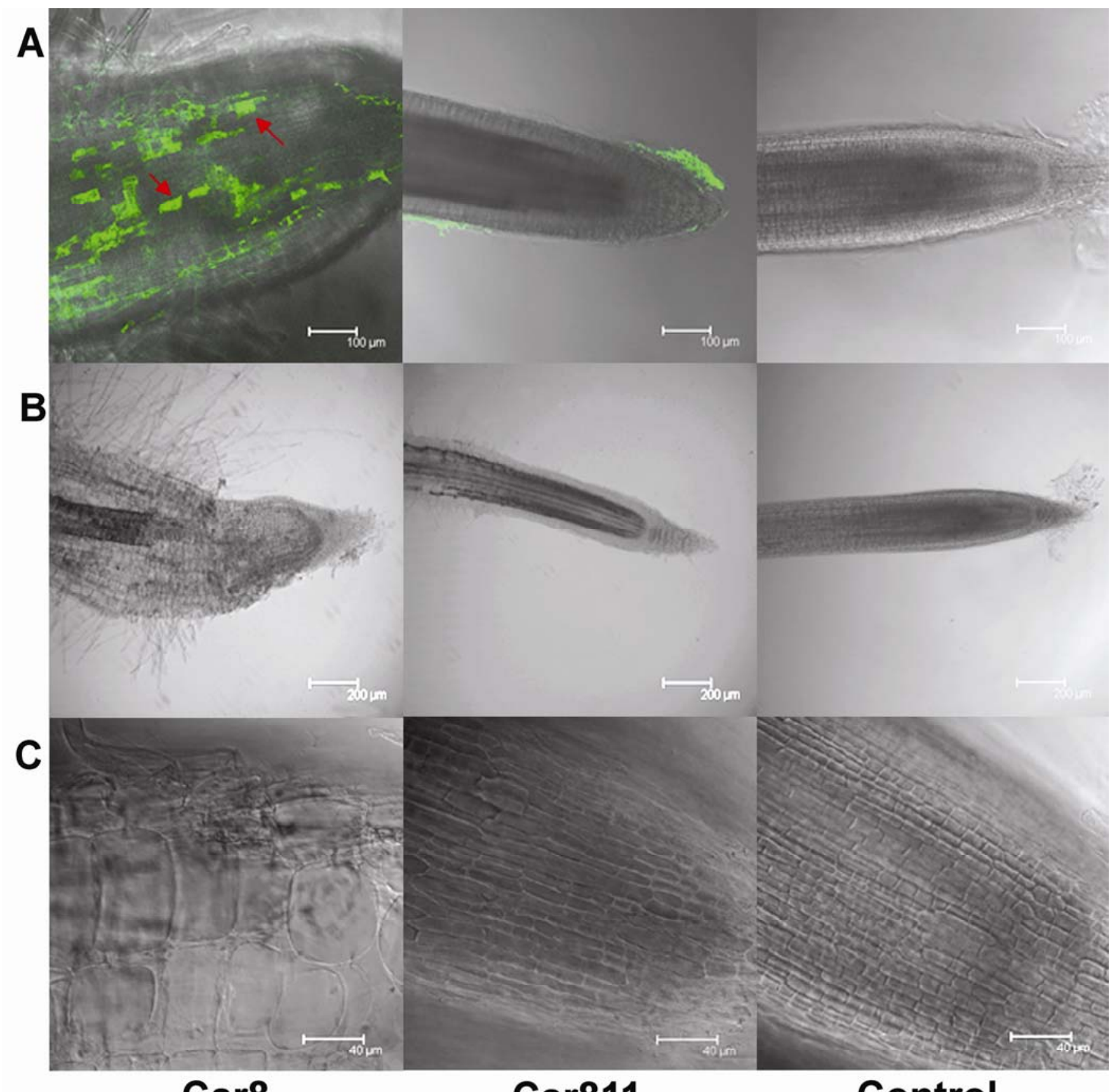

\section{Car8}

Fig. 7. Micrographs of Radish root tips colonized by green fluorescent protein (GFP)-labeled Streptomyces turgidiscabies strains. Radish plants (cv. Burpee White) were grown in a sterile hydroponic system at $22^{\circ} \mathrm{C}$ with a day length of $16 \mathrm{~h}$. Seedlings were inoculated with $10^{6}$ spores of GFP-labeled $S$. turgidiscabies strains. Tubes were incubated for 7 days with moderate shaking (approximately $100 \mathrm{rpm}$ ). Root tips of inoculated radish plants were mounted in water and observed using a Leica TCS SP2 microscope (Leica Microsystems). GFP was visualized using a four-line argon laser with an excitation wavelength of $488 \mathrm{~nm}$ and an emission wavelength of 500 to $600 \mathrm{~nm}$. Radish root tips were inoculated with S. turgidiscabies Car8, the $\Delta$ nec1 mutant Car811, or not inoculated. Magnification is indicated by the size bars which represent A, $100 \mu \mathrm{m}, \mathbf{B}, 200 \mu \mathrm{m}$, and $\mathbf{C}, 40 \mu \mathrm{m}$. 
growth was largely absent (Fig. 7). External colonization of root surfaces distal to the root tip by the two strains was extensive and indistinguishable, but not associated with necrosis (not shown).

\section{DISCUSSION}

The necl gene encodes a novel, secreted protein that has a dramatic virulence phenotype on seedlings in three plant genera (Figs. 5 to 7). Secreted virulence proteins are a common theme in bacterial pathogenesis (Byrne et al. 2001; Collmer et al. 2002); however, this is the first example of a secreted virulence protein in plant-pathogenic Streptomyces spp. Nec1 is secreted in the form of an N-terminal-processed, 16-kDa mature protein (Fig. 1). Two translational start sites (GTG and ATG-2) (Fig. 2) lie downstream of the transcriptional start sites $\mathrm{T} 1$ and $\mathrm{T} 2$ and each of the putative proteins encoded might travel by both the Sec and Tat pathway, based on bioinformatics analyses. Depending on the translational start site and the secretion pathway, the mature protein is predicted to begin at amino acids 83 or 90 . However, necl deletion analysis indicated that truncation at amino acid 81 inactivates Nec1, suggesting that cleavage sites were not accurately predicted by current algorithms (Bendtsen et al. 2004, 2005). Though the deletion analysis is consistent with an $\mathrm{N}$-terminal signal sequence, it does not allow us to infer the secretion pathway. Furthermore, some proteins travel both the Sec and Tat pathways in Streptomyces spp. (Schaerlaekens et al. 2004).

Regulation of expression of Nec1 appears to be fundamentally different than thaxtomin biosynthesis. The Nec1 protein is produced and secreted in CRM, a rich defined growth medium (Fig. 1A), whereas thaxtomin production requires a plant-based medium for production and is catabolically repressed (Babcock et al. 1993; Loria et al. 1995). Also, Nec1 production is initiated prior to $20 \mathrm{~h}$ of growth (Fig. 1A), whereas thaxtomin production is initiated after $48 \mathrm{hrs}$ (Babcock et al. 1993). These data suggest that the Nec1 protein is available to interact with host plant cells early in the succession of hostpathogen interactions, prior to the production of the phytotoxin thaxtomin. Transcription of nec1 does appear to be influenced by glucose, however. When $S$. turgidiscabies is grown in the thaxtomin-inducing medium $\mathrm{OBB}$, necl transcription originates from one site, T2; however, when this medium is amended with glucose, transcription occurs at both T1 and T2 (Figs. 2 and 3). The presence of direct repeats flanking T1 suggests the involvement of a transcriptional repressor that regulates the transcription of necl from T1 in response to glucose. Alternative transcriptional start sites function in gene transcriptional regulation in other Streptomyces spp. (Bignell et al. 2005; (McDowall et al. 1999; Wang et al. 2004). In the case of $n e c 1$, activation of an additional transcriptional start site may result in upregulation of gene expression in response to glucose. We speculate that necl is upregulated and has a critical role early in the infection process. Small amounts of glucose are likely available adjacent to the root as the result of cell wall modification during cell division and expansion, which might be sufficient to upregulate necl. This is consistent with the fact that thaxtomin inhibits cell wall biosynthesis (Scheible et al. 2003), and that the pathogen invades actively growing tissues (Loria et al. 1997). It is likely that other genes involved in the early stages of infection also are upregulated by glucose or other products of cell wall synthesis and modification.

Plant-pathogenic Streptomyces spp. that cause potato scab are neither host nor tissue specific. Scab lesions are produced on potato tubers, which are modified, underground stems, as well on taproots, including carrot, beet, and radish (Loria et al. 1997). S. scabies even causes a scab-like lesion on peanut shell, which is pericarp tissue (Kritzman et al. 1996). Seedlings of both monocot and dicot plants also are infected by scab-causing Streptomyces spp. (Hooker 1949; Leiner et al. 1996). These infections result in root necrosis, plant stunting, and death; an absence of lateral expansion of these tissues precludes the formation of scab lesions. The lack of host and tissue specificity of these pathogens suggests that their pathogenicity strategies target components of cells that are conserved across plant families and expressed in multiple plant tissues. The activity of thaxtomin on monocot and dicot seedlings is consistent with this model (Hooker 1949; Leiner et al. 1996). The target of thaxtomin is not known; however, experiments conducted with Arabidopsis seedlings demonstrate that cellulose synthesis, a highly conserved process in higher plants, is inhibited by the toxin (Scheible et al. 2003). Like thaxtomin, Nec1 appears to have a highly conserved plant target. Indeed, the Nec1 protein caused necrosis on excised tuber tissue and contributed greatly to the virulence of $S$. turgidiscabies Car8 on Arabidopsis, tobacco, and radish (Figs. 5 to 7).

The virulence protein Nec1 appears to be required for full virulence of $S$. turgidiscabies on different plant species. Plants inoculated with the $\Delta n e c 1$ mutant showed some root necrosis and were slowed in growth, relative to the control. However, most plants did survive and appeared to recover from the infection. In contrast, all radish and Arabidopsis plants inoculated with the wild-type strain were severely necrotic, and most died over the course of the 3-week experiment. Though many tobacco plants inoculated with the wild-type strain survived, root necrosis was extensive and the plants never grew out of the infection. Thaxtomin production does not depend on necl; therefore, this gene is an independent virulence factor that appears to be regulated differently than is thaxtomin. The role of the Nec1 protein is not known. One possibility is that the plant defense response was more successful in plants inoculated with the $\Delta n e c 1$ mutant than in those inoculated with the wild-type strain. There are examples of secreted proteins that act to suppress plant defense responses (DebRoy et al. 2004; He et al. 2006).

Tobacco plants inoculated with both the wild-type $S$. turgidiscabies Car8 and the $\Delta n e c 1$ mutant produced gall-like structures that developed from the infected root tips. Anatomical studies were not done to determine whether these gall-like structures were organized. However, production of either galls or neoplastic growth involves cell proliferation and modification which require both cytokinin and auxin (Vandeputte et al. 2005). The $S$. turgidiscabies PAI contains a putative cytokinin biosynthetic operon (Kers et al. 2005) and auxin production by plant-pathogenic Streptomyces spp. has been demonstrated (Manulis et al. 1994). Gall-like structures were very frequent and quite necrotic on root tips infected with $S$. turgidiscabies Car8. In contrast, roots infected with the $\Delta n e c 1$ mutant had fewer and smaller galls and less necrosis. Production of roots and shoots from the gall-like structures in plants inoculated with the $\Delta n e c 1$ mutant suggests that the plant was able to out grow the infection when the Nec1 protein was not present.

Detailed examination of radish roots inoculated with GFPlabeled $S$. turgidiscabies strains demonstrates that necl fundamentally alters the very early interactions of this pathogen with the host (Fig. 7). Aggressive colonization of the internal and external tissues of the root meristem by $S$. turgidiscabies resulted in radial expansion of cells, typical of the response described for thaxtomin (Fry and Loria 2002; Scheible et al. 2003). Abnormal and prolific production of root hairs in the zone of differentiation in wild-type infected plants could be the result of increased levels of auxin in the meristematic region, because this is a documented auxin response (Rahman et al. 2002); however, this phenotype is not diagnostic of exces- 
sive auxin production and may be due to other factors. In contrast, there was almost no root meristem colonization by the $\Delta n e c 1$ mutant, and the occasional colonies were limited to the root surface. Roots were slightly swollen relative to the controls and aberrant root hair production was minimal, suggesting that distortion of the root morphology in plants inoculated by the wild-type pathogen was due to internal colonization and secretion of virulence factors, such as thaxtomin and possibly auxin and cytokinin, inside plant tissue. The milder symptoms produced by $S$. turgidiscabies Car811 on root tips appeared to be due to very minimal external colonization, resulting in lower exposure of the meristematic region to secreted virulence factors.

The lack of homologs and recognizable motifs in Nec1 makes it difficult to predict function; however, based on deletion analyses the region of the Nec1 protein required for activity lies in the N-terminal 151 amino acids of the protein. The bioassays suggest that the necl gene is required for colonization of the root meristem, which appears to be a prerequisite for maximal disease development. The lack of an effective plant defense response in wild-type inoculated plants, and the apparent success of plant defense mechanisms in the absence of Nec1, suggests that the role of this protein may be to suppress the host defense response, as occurs in other systems (Janjusevic et al. 2006; Rooney et al. 2005).

Thaxtomin is the only other documented pathogenicity determinant in this host-pathogen system (Healy et al. 2000), and is known to inhibit cellulose synthesis in expanding and dividing plant cells (Scheible et al. 2003). Activity of this toxin on the cell wall integrity of expanding cells is consistent with the fact that scab-producing streptomycetes infect only meristematic regions of potato tubers (Hooker and Page 1960). Thaxtomin has been reported to induce a subset of plant defense responses in Arabidopsis. The toxin stimulates a rapid $\mathrm{Ca}^{2+}$ influx, followed by a net $\mathrm{H}^{+}$efflux across the plasma membrane of root cells, which is most pronounced in the zone of elongation (Tegg et al. 2005). Thaxtomin also induced apoptosis in Arabidopsis suspension-cultured cells (Duval et al. 2005). It may be that a role for Nec1 is to suppress plant defenses stimulated by thaxtomin. It is interesting to note that, even though thaxtomin is a highly active toxin that is required for plant pathogenicity, it is not sufficient for meristem infection and maximal disease development.

If Nec1 does suppress plant defense responses, it likely would need to be delivered into the plant cell cytoplasm. Our data demonstrate that the Nec1 protein is secreted from Streptomyces cells, apparently through the Sec or Tat pathways, but does not reveal how Nec1 might enter plant cells. Our results confirm that infection is through the meristematic region in roots, as it is in potato tubers (Hooker 1950). It is possible that these filamentous pathogens penetrate nescient walls in newly divided cells and secrete Nec1 directly into the cell. Plant-pathogenic Streptomyces spp. are capable of penetrating plant cell walls (Clark and Matthews 1987). Once inside the cell wall, Nec1 might move into the cytoplasm through active uptake, diffusion, or endocytosis. Because Nec1 has a strong pathogenicity phenotype and lacks homologs in public databases, it qualifies as an entirely novel virulence protein and may reveal previously undescribed strategies for manipulation of plants by microbes.

\section{MATERIALS AND METHODS}

Strains, plasmids, and culture conditions.

S. turgidiscabies Car8 has been described previously (Kers et al. 2004, 2005). The International Streptomyces Project Medium 2 (ISP2) and ISP4 (BD Biosciences, San Jose, CA, U.S.A.) were used for routine cultivation of Streptomyces strains. MSF medium was used in Nec1 secretion studies. Spore suspensions of $S$. turgidiscabies Car8 strains were prepared as previously described (Kieser et al. 2000). For virulence assays on Arabidopsis, radish, and tobacco, inoculum of $S$. turgidiscabies strains were grown for 2 days in OBB (Goyer et al. 1998). DNA was extracted from Streptomyces cultures grown in CRM medium (Pigac and Schrempf 1995) or YEME medium (Kieser et al. 2000 ) for 24 to $48 \mathrm{~h}$ at $30^{\circ} \mathrm{C}$ using a modification of the procedure of Rao and associates (Rao et al. 1987).

GFP-labeled strains of $S$. turgidiscabies Car8 and the corresponding $\Delta n e c 1$ strain Car811 were constructed to study the colonization by using confocal laser microscopy. Both strains were transformed with an 8.0-kb vector, pIJ8641 (Sun et al. 1999), carrying the egfp gene downstream of a strong constitutive ErmE promoter, and an apramycin-resistant marker. The strain has been replicated in the methylation-deficient $E$. coli strain ET12567 containing the helper plasmid pUZ8002 prior to conjugation into $S$. turgidiscabies.

Cloning and DNA manipulations were done in E. coli DH5 $\alpha$ using pBluescript II (Stratagene, La Jolla, CA, U.S.A.) following standard procedures. For the heterologous expression of $n e c 1$, we used pET28a (Novagen), an E. coli T7 polymerasedependent expression vector. The $E$. coli strains BLR (DE3) (Novagen) and ET12567 (MacNeil 1987) were used for T7 induction and for propagation of demethylated plasmid DNA for transformation experiments, respectively.

\section{Nec1 expression and analysis in $E$. coli.}

A restriction site-modified primer pair was designed to polymerase chain reaction (PCR) amplify the full-length necl gene as previously described (Bukhalid et al. 1998). The resulting 0.6-kb PCR product was digested with EcoRI and NdeI and ligated into pET28a (pET::necl). Deletion constructs were made by PCR amplification using specific primer pairs designed to amplify the DNA fragment from the necl gene and ligated into pET28a (Table 1). The ligated product was first transformed to DH5 $\alpha$ to facilitate sequencing and a correct clone was transformed to BLR(DE3). Exponentially growing cultures (optical density at $600 \mathrm{~nm}$ of 0.5 ) of E. coli BLR (DE3) containing pET::necl or a vector control were induced with $1 \mathrm{mM}$ IPTG. Total proteins were separated by SDSPAGE using standard procedures (Sambrook et al. 1989).

To investigate the role of the Nec1 protein in the necrogenic phenotype on potato tuber tissue, the necl gene product expressed in $E$. coli was subjected to protease or heat inactivation as follows. A $100-\mu l$ aliquot of induced cultures of $E$. coli BLR(DE3) pET::necl and vector control were pelleted, and resuspended in $10 \mathrm{mM}$ MES (morpholineethanesulfonic acid) ( $\mathrm{pH}$ 5.8). Cells were sonicated and pelleted to remove the insoluble fraction, and the supernatant was subjected to heat and Pronase (EMD Biosciences, San Diego, CA, U.S.A.) sensitivity experiments as follows. Equal volumes $(50 \mu \mathrm{l})$ were exposed to either 95 or $30^{\circ} \mathrm{C}$ for $10 \mathrm{~min}$ or incubated at $37^{\circ} \mathrm{C}$ for $30 \mathrm{~min}$ in the presence or absence of Pronase at $0.5 \mathrm{mg} / \mathrm{ml}$. Necrogenic activity was detected in the EPT assay.

Western analysis of the Nec1 protein was accomplished by separating proteins with SDS-PAGE and transferring to Immobilon-P polyvinylidene difluoride membranes (Bio-Rad Laboratories, Hercules, CA, U.S.A.) using the Trans-Blot SD semidry electrophoretic transfer cell (Bio-Rad). Nec1 was detected with Nec1-specific rabbit polyclonal antibody, followed by goat anti-rabbit immunoglobulin G-alkaline phosphate conjugate (Sigma-Aldrich, St. Louis). Membrane-bound secondary antibodies were visualized by chemiluminescence using the horseradish peroxidase-based ECL Western Blotting detection system (Amersham Biosciences, Piscataway, NJ, U.S.A.). 


\section{Disruption, complementation, and expression} of nec1 in S. turgidiscabies.

A necl-deficient mutant of strain S. turgidiscabies Car8, generated by marker exchange mutagenesis, was described previously (Kers et al. 2005). A complementation construct was designed using the Streptomyces expression vector pSET152. A SacII-digested 1,552-bp fragment of cosmid DNA, containing necl, was ligated into the SacII site of pSET152 $\left(\right.$ apra $\left.^{\mathrm{R}}\right)$. Transformed colonies of $E$. coli DH5 $\alpha$ were selected with apramycin. The pSET152::necl construct then was propagated in E. coli ET12567 and conjugated to $S$. turgidiscabies C811. Colony PCR was performed on apramycinresistant transformants, and PCR products were sequenced to confirm a mutation-free insert.

Cultures of $S$. turgidiscabies Car8 were grown in CRM at $30^{\circ} \mathrm{C}$. At 5-h time intervals, $10-\mathrm{ml}$ aliquots were removed and separated into cell pellets and supernatant fractions by centrifugation. Then, $100 \mathrm{mM}$ PMSF (Sigma-Aldrich) was added to each supernatant fraction to inhibit protease activity and the supernatant was filter sterilized by passing through a $0.2-\mu \mathrm{m}$ filter. Proteins in the supernatants were concentrated 50 -fold by precipitation with $20 \%$ TCA and resuspended in gel loading buffer. Proteins from cell pellet and supernatant fractions were resolved by SDS-PAGE and analyzed by immunoblotting with anti-Nec1 polyclonal antibody produced in rabbits.

\section{Transcriptional mapping and bioinformatics analyses.}

$S$. turgidiscabies culture was grown in OBB medium for 3 days, with or without $10 \%$ glucose, and total RNA was extracted using the modified Kirby method (Kieser et al. 2000). For S1 nuclease reactions, $40 \mu \mathrm{g}$ of RNA was hybridized with a 323-bp radiolabeled probe in sodium trichloroacetate buffer at $45^{\circ} \mathrm{C}$ for 12 to $14 \mathrm{~h}$. The transcription start site of necl was determined by this 323-bp probe generated by PCR using oligonucleotide MJ91 (5'-CAACCACGGCGGGAGC) and MJ 92 (5'-GCTTTGGCGAACTTGATTGAG). Antisense primer MJ92, which anneals within the necl coding region, was labeled at its $5^{\prime}$ end with $\left[{ }^{32} \mathrm{P}\right] \mathrm{dATP}$ using $\mathrm{T} 4$ polynucleotide kinase and used with the unlabeled sense primer MJ91, which anneals upstream of the necl gene. A DNA sequencing ladder was generated using a DNA dideoxyterminal sequencing kit (USB Corp., Cleveland, OH, U.S.A.) with the same labeled primer MJ92 as was used for the probe.

The $5^{\prime}$ end of the cDNA-encoding necl gene was isolated by the RACE protocol (BD Biosciences Clontech, Palo Alto, CA, U.S.A.). Cultures were grown and RNA was extracted as described previously. For each culture condition, $340 \mathrm{ng}$ of total RNA was used to synthesize first-strand cDNA, as described by the manufacturer, using a gene-specific primer corresponding to the complement of the sequence at base pair positions 237 to 262. An oligonucleotide (BD SMART II, 5'-AAGCAG TGGTATCAACGCAGAGTACGCGGG-3') contains a terminal stretch of $\mathrm{G}$ residues, which anneal to the $\mathrm{dC}$-rich cDNA tail and serves as an extended template for reverse transcription. BD PowerScript RT switches templates from the mRNA molecule to the BD SMART oligo, generating a complete 5' cDNA copy of the original RNA with the additional BD SMART sequence at the end. The anchored cDNAs were amplified with a BD SMART oligo-specific sense primer and gene-specific antisense primer. The resulting $5^{\prime}$ RACE product was isolated by agarose gel electrophoresis and purified from the gel using the QIAquick Gel Extraction Kit (Qiagen, Valencia, CA, U.S.A.) and sequenced.

The DNA sequence upstream of the necl coding region was analyzed for bacterial sigma 70 promoters using BPROM as well as manually searched for streptomycete promoters (Strohl
1992). Protein secretion signals and cleavage sites were predicted with SignalP 3.0 and TatP 1.0.

\section{Plant production and bioassays.}

Excised potato tuber slices were used to assay Nec1-induced necrosis; this technique has been described previously (Loria et al. 1995). Potato (cv. Chippewa) tubers were surface disinfested and a core of tissue was removed from the center of the tuber. Discs of tissue were aseptically sliced from the core and placed on moist filter paper in a petri plate. Protein extracts from cell cultures or crude cell lysates were applied to the disk and then were incubated in the dark at room temperature for 3 to 5 days.

For virulence assays, A. thaliana (ecotype Columbia), N. tobacum, and radish (cv. Burpee White; W. Atlee Burpee \& Company, Warminster, PA, U.S.A.) plants were produced in vitro from seed on Murashige and Skoog agar medium (MS) with $2 \%$ sucrose (Murashige and Skoog 1962) in Magenta boxes. Plants were grown at $21 \pm 2^{\circ} \mathrm{C}$ with a 16 -h day length and were inoculated with Streptomyces wild-type and mutant strains after 7 days. Bacterial cultures were grown for 2 days in OBB (Goyer et al. 1998) medium prior to adding to the surface of the MS medium in the vicinity of the seedling roots. After 21 to 28 days, plants were removed from the boxes and media was rinsed from the roots for observation of disease symptoms.

Radish plants (cv. Burpee White) produced for confocal laser microscopy were grown in a sterile hydroponic system using $15 \mathrm{ml}$ of $\mathrm{MS}$ salts solution (half-strength, SigmaAldrich) in 25-by-150-mm glass tubes (Bellco Biotechnology, Vineland, NJ U.S.A.). Filter paper (Whatman no. $1,9 \mathrm{~cm}$ in diameter) was folded over a smaller diameter tube and pushed into the hydroponics tube, with the flat surface of the filter paper sitting just above the level of the liquid. Surface-sterile seed were placed atop the filter paper and tubes incubated at $4^{\circ} \mathrm{C}$ for $48 \mathrm{~h}$. Tubes then were incubated at $22^{\circ} \mathrm{C}$ with a day length of $16 \mathrm{~h}$. Seedlings were inoculated with $10^{6}$ spores of GFP-labeled S. turgidiscabies strains in $100 \mu \mathrm{l}$ of $0.9 \% \mathrm{NaCl}$. Control tubes were inoculated with $100 \mu \mathrm{l}$ of $0.9 \% \mathrm{NaCl}$. The inoculum was applied down the inside of the tube directly into the media solution. Tubes were incubated for 7 days with moderate shaking (approximately $100 \mathrm{rpm}$ ). Root tips of inoculated radish plants were mounted in water and observed using a Leica TCS SP2 microscope (Leica Microsystems, Wetzlar, Germany). GFP was visualized using a four-line argon laser with an excitation wavelength of $488 \mathrm{~nm}$ and an emission wavelength of 500 to $600 \mathrm{~nm}$.

\section{ACKNOWLEDGMENTS}

This project was supported by the National Research Initiative of the USDA Cooperative State Research, Education and Extension Service, grant number 2005-35319-15289.

\section{LITERATURE CITED}

Babcock, M. J., Eckwall, E. C., and Schottel, J. L. 1993. Production and regulation of potato-scab-inducing phytotoxins by Streptomyces scabies. J. Gen. Microbiol. 139 (7):1579-1586.

Belfaquih, N., Jaspers, C., Kurzatkowski, W., and Penninckx, M. J. 2002. Properties of Streptomyces sp endo-beta-xylanases in relation to their applicability in kraft pulp bleaching. World J. Microb. Biot. 18(7):699705.

Bendtsen, J. D., Nielsen, H., von Heijne, G., and Brunak, S. 2004. Improved prediction of signal peptides: SignalP 3.0. J. Mol. Biol. 340(4):783-795.

Bendtsen, J. D., Nielsen, H., Widdick, D., Palmer, T., and Brunak, S. 2005. Prediction of twin-arginine signal peptides. BMC Bioinformatics 6:167.

Bentley, S. D., Chater, K. F., Cerdeno-Tarraga, A. M., Challis, G. L., 
Thomson, N. R., James, K. D., Harris, D. E., Quail, M. A., Kieser, H., Harper, D., Bateman, A., Brown, S., Chandra, G., Chen, C. W., Collins, M., Cronin, A., Fraser, A. Goble, A., Hidalgo, J., Hornsby, T., Howarth, S., Huang, C. H., Kieser, T., Larke, L. Murphy, L., Oliver, K., O’Neil, S., Rabbinowitsch, E., Rajandream, M. A., Rutherford, K., Rutter, S., Seeger, K., Saunders, D., Sharp, S., Squares, R., Squares, S., Taylor, K., Warren, T., Wietzorrek, A., Woodward, J., Barrell, B. G., Parkhill, J., and Hopwood, D. A. 2002. Complete genome sequence of the model actinomycete Streptomyces coelicolor A3(2). Nature 417(6885):141-147.

Bignell, D. R., Tahlan, K. R. Colvin, K. R., Jensen, S. E., and Leskiw, B. K. 2005. Expression of $c c a R$, encoding the positive activator of cephamycin C and clavulanic acid production in Streptomyces clavuligerus, is dependent on bldG. Antimicrob. Agents Ch. 49(4):1529-1541.

Broadway, R. M., Williams, D. L., Kain, W. C., Harman, G. E., and Lorito, M. 1995. Partial characterization of chitinolytic enzymes from Streptomyces albidoflavus. Lett. Appl. Microbiol. 20(5):271-276.

Bukhalid, R. A., and Loria, R. 1997. Cloning and expression of a gene from Streptomyces scabies encoding a putative pathogenicity factor. J. Bacteriol. 179(24):7776-7783.

Bukhalid, R. A., Chung, S. Y., and Loria, R. 1998. necl, a gene conferring a necrogenic phenotype, is conserved in plant-pathogenic Streptomyces spp. and linked to a transposase pseudogene. Mol. Plant-Microbe Interact. 11(10):960-967.

Bukhalid, R. A., Takeuchi, T., Labeda, D., and Loria, R. 2002. Horizontal transfer of the plant virulence gene, necl, and flanking sequences among genetically distinct Streptomyces strains in the Diastatochromogenes cluster. Appl. Environ. Microbiol. 68(2):738-744.

Byrne, B. A., Prescott, J. F., Palmer, G. H., Takai, S., Nicholson, V. M., Alperin, D. C., and Hines, S. A. 2001. Virulence plasmid of Rhodococcus equi contains inducible gene family encoding secreted proteins. Infect. Immun. 69(2):650-656.

Challis, G. L., and Hopwood, D. A. 2003. Synergy and contingency as driving forces for the evolution of multiple secondary metabolite production by Streptomyces species. Proc. Natl. Acad. Sci. U.S.A. 100(Suppl. 2):14555-14561.

Clark, C. A., and Matthews, S. W. 1987. Histopathology of sweet potato root infection by Streptomyces ipomoea. Phytopathology 77(10):14181423.

Collmer, A., Lindeberg, M., Petnicki-Ocwieja, T., Schneider, D. J., and Alfano, J. R. 2002. Genomic mining type III secretion system effectors in Pseudomonas syringae yields new picks for all TTSS prospectors. Trends Microbiol. 10(10):462-469.

DebRoy, S., Thilmony, R., Kwack, Y. B., Nomura, K., and He, S. Y. 2004. A family of conserved bacterial effectors inhibits salicylic acid-mediated basal immunity and promotes disease necrosis in plants. Proc. Natl. Acad. Sci. U.S.A. 101(26):9927-9932.

Duval, I., Brochu, V., Simard, M., Beaulieu, C., and Beaudoin, N. 2005. Thaxtomin A induces programmed cell death in Arabidopsis thaliana suspension-cultured cells. Planta 222(5):820-831.

Fry, B. A., and Loria, R. 2002. Thaxtomin A: evidence for a plant cell wall target. Physiol. Mol. Plant Pathol. 60:1-8.

Gauthier, C., Li, H., and Morosoli, R. 2005. Increase in xylanase production by Streptomyces lividans through simultaneous use of the Sec- and Tat-dependent protein export systems. Appl. Environ. Microbiol. 71(6):3085-3092.

Goyer, C., Vachon, J., and Beaulieu, C. 1998. Pathogenicity of Streptomyces scabies mutants altered in thaxtomin A production. Phytopathology. 88 5):442-445.

He, P., Shan, L., Lin, N. C., Martin, G. B., Kemmerling, B., Nurnberger, T., and Sheen, J. 2006. Specific bacterial suppressors of MAMP signaling upstream of MAPKKK in Arabidopsis innate immunity. Cell 125(3):563-575

Healy, F. G., Wach, M., Krasnoff, S. B., Gibson, D. M., and Loria, R. 2000. The $t x t A B$ genes of the plant pathogen Streptomyces acidiscabies encode a peptide synthetase required for phytotoxin thaxtomin A production and pathogenicity. Mol. Microbiol. 38:794-804.

Hooker, W. J. 1949. Parasitic action of Streptomyces scabies on roots of seedlings. Phytopathology 39:442-462.

Hooker, W. J. 1950. A technique for observing tuber enlargement and scab development in potatoes. Phytopathology 40:390-391.

Hooker, W. J., and Page, O. T. 1960. Relation of potato tuber growth and skin maturity to infection by common scab, Streptomyces scabies. Am. Potato J. 37:414-423.

Ikeda, H., Ishikawa, J., Hanamoto, A., Shinose, M., Kikuchi, H., Shiba, T., Sakaki, Y., Hattori, M., and Omura, S. 2003. Complete genome sequence and comparative analysis of the industrial microorganism Streptomyces avermitilis. Nat. Biotechnol. 21(5):526-531.

Janjusevic, R., Abramovitch, R. B., Martin, G. B., and Stebbins, C. E. 2006. A bacterial inhibitor of host programmed cell death defenses is an E3 ubiquitin ligase. Science 311(5758):222-226.
Kaltenpoth, M., Gottler, W., Herzner, G., and Strohm, E. 2005. Symbiotic bacteria protect wasp larvae from fungal infestation. Curr. Biol. 15(5):475-479.

Kers, J. A., Wach, M. J., Krasnoff, S. B., Widom, J., Cameron, K. D., Bukhalid, R. A., Gibson, D. M., Crane, B. R., and Loria, R. 2004. Nitration of a peptide phytotoxin by bacterial nitric oxide synthase. Nature 429(6987):79-82.

Kers, J. A., Cameron, K. D., Joshi, M. V., Bukhalid, R. A., Morello, J. E., Wach, M. J., Gibson, D. M., and Loria, R. 2005. A large, mobile pathogenicity island confers plant pathogenicity on Streptomyces species. Mol. Microbiol. 55(4):1025-1033.

Kieser, T., Bibb, M. J. Buttner, M. J., Chater, K. F., and Hopwood, D. A. 2000. Practical Streptomyces Genetics. The John Innes Foundation, Norwich, U.K.

King, R. R., Lawrence, C. H., Clark, M. C., and Calhoun, L. A. 1989. Isolation and characterization of phytotoxins associated with Streptomyces scabies. J. Chem. Soc. Chem. Commun. 13:849-850.

King, R. R., Lawrence, C. H., and Calhoun, L. A. 1996. Isolation and identification of pigments generated in vitro by Streptomyces acidiscabies. J. Agric. Food Chem. 44(9):2849-2851.

Kritzman, G., Shani, C. A., Kirshner, B., Riven, Y., Bar, Z., Katan, J., and Grinstein, A. 1996. Pod wart disease of peanuts. Phytoparasitica 24(4):293-304.

Leiner, R. H., Fry, B. A., Carling, D. E., and Loria, R. 1996. Probable involvement of thaxtomin A in pathogenicity of Streptomyces scabies on seedlings. Phytopathology 86(7):709-713.

Loria, R., Bukhalid, R. A., Creath, R. A., Leiner, R. H., Olivier, M., and Steffens, J. C. 1995. Differential production of thaxtomins by pathogenic Streptomyces species in vitro. Phytopathology 85(5):537-541.

Loria, R., Bukhalid, R. A., Fry, B. A., and King, R. R. 1997a. Plant pathogenicity in the genus Streptomyces. Plant Dis. 81(8):836-846.

MacNeil, D. J. 1987. Introduction of plasmid DNA into Streptomyces lividans by electroporation. FEMS (Fed. Eur. Microbiol. Soc.) Microbiol. Lett. 42:239-244.

Manulis, S., Shafrir, H., Epstein, E., Lichter, A., and Barash, I. 1994. Biosynthesis of indole-3-acetic acid via the indole-3-acetamide pathway in Streptomyces spp. Microbiology 140(Pt. 5):1045-1050.

McDowall, K. J., Thamchaipenet, A., and Hunter, I. S. 1999. Phosphate control of oxytetracycline production by Streptomyces rimosus is at the level of transcription from promoters overlapped by tandem repeats similar to those of the DNA-binding sites of the OmpR family. J. Bacteriol. 181(10):3025-3032.

Murashige, T, and Skoog, F. 1962. A revised medium for rapid growth and bioassays with tobacco tissue cultures. Physiol. Plant 15:473-497.

Parajuli, N., Basnet, D. B., Lee, H. C., Sohng, J. K., and Liou, K. 2004 Genome analyses of Streptomyces peucetius ATCC 27952 for the identification and comparison of cytochrome P450 complement with other Streptomyces. Arch. Biochem. Biophys. 425(2):233-241.

Pigac, J., and Schrempf, H. 1995. A simple and rapid method of transformation of Streptomyces rimosus R6 and other streptomycetes by electroporation. Appl. Environ. Microbiol. 61(1):352-356.

Rahman, A., Hosokawa, S., Oono, Y., Amakawa, T., Goto, N., and Tsurumi, S. 2002. Auxin and ethylene response interactions during Arabidopsis root hair development dissected by auxin influx modulators. Plant Physiol. 130(4):1908-1917.

Rao, R. N., Richardson, M. A., and Kuhstoss, S. 1987. Cosmid shuttle vectors for cloning and analysis of Streptomyces DNA. Methods Enzymol 153:166-198

Robison, K., McGuire, A. M., and Church, G. M. 1998. A comprehensive library of DNA-binding site matrices for 55 proteins applied to the complete Escherichia coli K-12 genome. J. Mol. Biol. 284(2):241254.

Rooney, H. C., Van't Klooster, J. W., van der Hoorn, R. A., Joosten, M. H., Jones, J. D., and de Wit, P. J. 2005. Cladosporium Avr2 inhibits tomato $\mathrm{Rcr} 3$ protease required for $\mathrm{Cf}-2$-dependent disease resistance. Science 308(5729):1783-1786.

Sambrook, J., Fritsch, E. F., and Maniatis, T. 1989. Molecular Cloning: A Laboratory Manual. Cold Spring Harbor Laboratory Press, Cold Spring Harbor, NY, U.S.A.

Schaerlaekens, K., Lammertyn, E., Geukens, N., De Keersmaeker, S. Anne, J., and Van Mellaert, L. 2004. Comparison of the Sec and Tat secretion pathways for heterologous protein production by Streptomyces lividans. J. Biotechnol. 112(3):279-288.

Scheible, W. R., Fry, B., Kochevenko, A., Schindelasch, D., Zimmerli, L., Somerville, S., Loria, R., and Somerville, C. R. 2003. An Arabidopsis mutant resistant to thaxtomin A, a cellulose synthesis inhibitor from Streptomyces species. Plant Cell 15(8):1781-1794.

Strohl, W. R. 1992. Compilation and analysis of DNA sequences associated with apparent streptomycete promoters. Nucleic Acids Res. 20(5):961-974 
Tegg, R. S., Melian, L., Wilson, C. R., and Shabala, S. 2005. Plant cell growth and ion flux responses to the streptomycete phytotoxin thaxtomin A: calcium and hydrogen flux patterns revealed by the non-invasive MIFE technique. Plant Cell Physiol. 46(4):638-648.

Tokala, R. K., Strap, J. L., Jung, C. M., Crawford, D. L., Salove, M. H., Deobald, L. A., Bailey, J. F., and Morra, M. J. 2002. Novel plantmicrobe rhizosphere interaction involving Streptomyces lydicus WYEC108 and the pea plant (Pisum sativum). Appl. Environ. Microbiol. 68(5):2161-2171.

Vandeputte, O., Oden, S., Mol, A., Vereecke, D., Goethals, K., El Jaziri, M., and Prinsen, E. 2005. Biosynthesis of auxin by the gram-positive phytopathogen Rhodococcus fascians is controlled by compounds spe- cific to infected plant tissues. Appl. Environ. Microbiol. 71(3):11691177.

Wang, L., Tahlan, K., Kaziuk, T. L., Alexander, D. C., and Jensen, S. E. 2004. Transcriptional and translational analysis of the $c c a R$ gene from Streptomyces clavuligerus. Microbiology 150(Pt. 12):4137-4145.

\section{AUTHOR-RECOMMENDED INTERNET RESOURCES}

SignalP 3.0 server: www.cbs.dtu.dk/services/SignalP Softberry website: www.softberry.com

TatP 1.0 server: www.cbs.dtu.dk/services/TatP 